\title{
Investigation of LTE Receivers in Term of Channel Equalization Techniques in LTE Downlink
}

\author{
Ibrahim A. Alfatah Ibrahim ${ }^{1}$, Hala E I Jubara ${ }^{2}$ \\ ${ }^{1,2}$ Alnielian University, Department of Engineering, Khartoum, Sudan
}

\begin{abstract}
In this paper we will introduce a comprehensive study based on LTE techniques that are used in order to eliminate or reduce different types of interferences that happens in the wireless communication channel and affect the received signal at receivers which associated with channel equalization techniques by comparing between three different techniques which are the Zero Forcing ZF, the Minimum Mean Square Error MMSE and the Soft Sphere Decoding SSD. Also different types of modulations are considered such as QPSK, 16QAM, and 64QAM. The comparison is performed for different transmission modes such as SISO, SIMO, and MIMO. And the results show that for SISO and MIMO the MMSE has the advantage over the ZF equalizer, and in SIMO the ZF is performing better than the MMSE, and finally for the MIMO we figure that the SSD equalizer has advantage over both other equalizers.
\end{abstract}

Keywords: LTE, Downlink, Equalization, ZF, MMSE, SSD, SISO, SIMO, MIMO.

\section{Introduction}

As the rapidly increase of the wireless communication technologies happens these days it become the most desirable field of study for researchers, and specifically for electronics and communication engineers and specialist. So that makes it very difficult and complex for exploration and discovery without virtual implementation or testing environments and the proper latest references of which criteria has been developed.

With the increase of demand on the multimedia services, 3GPP has proposed its long term evolution (LTE) system. The most significant improvement of LTE system is that it employs many novel air interface techniques. Accordingly exact channel estimation technology is the key to the high transmission performance and ensures the superiority of the system. So this paper is engaged in the research on the channel equalization technology for LTE downlink [1].

\section{Literature Review}

In OFDM, as well as SC-FDMA, equalization is achieved on the receiver side, after the FFT calculation, by multiplying each Fourier coefficient by a complex number. Thus, frequency selective fading and phase distortion can be easily combated. The advantage is that frequency domain equalization using FFTs requires less computation than conventional time domain equalization [2].

Equalization is one of the procedures used to reduce the ISI phenomenon by using a certain mathematical algorithm that predesigned and prototyped in the receiver physical implementation for this mission, the other way is by using the error correcting codes which known as turbo codes [3].

\subsection{Orthogonal Frequency-Division Multiplexing}

In recent years Orthogonal Frequency-Division Multiplexing (OFDM) has emerged as a successful air-interface technique $[12,13]$. In the context of wired environments, OFDM techniques are also known as Discrete Multi-Tone (DMT) transmissions and employed within Asymmetric Digital
Subscriber Line (ADSL), High-bit-rate Digital Subscriber Line (HDSL), and Very-high-speed Digital Subscriber Line (VDSL) [14]

OFDM has been advocated by many European standards, such as Digital Audio Broadcasting (DAB), Digital Video Broadcasting for Terrestrial television (DVB-T), Digital Video Broadcasting for Handheld terminals (DVB-H), Wireless Local Area Networks (WLANs) and Broadband Radio Access Networks (BRANs) [14]. Furthermore, OFDM has been ratified as a standard or has been considered as a candidate standard by a number of standardization groups of the Institute of Electrical and Electronics Engineers (IEEE), such as the IEEE 802.11 and the IEEE 802.16 standard families [15].

\subsubsection{OFDM advantages \& disadvantages}

Besides its implementation flexibility, the low complexity required in transmission and reception as well as the attainable high performance render, OFDM is a highly attractive candidate for highdatarate communications over time-varying frequency selective radio channels. For example, in classic single carrier systems, complex equalizers have to be employed at the receiver for the sake of mitigating the Inter-Symbol Interference (ISI) introduced by multi-path propagation. By contrast, when using a cyclic prefix, OFDM exhibits a high resilience against the ISI. Incorporating channel coding techniques into OFDM systems, which results in Coded OFDM (COFDM) [16], allows us to maintain robustness against frequency-selective fading channels, where busty errors are encountered at specific subcarriers in the FD.

Besides its significant advantages, OFDM also has a few disadvantages. One problem is the associated increased Peak-to-Average Power Ratio (PAPR) in comparison with single-carrier systems [13], requiring a large linear range for the OFDM transmitter's output amplifier. In addition, OFDM is sensitive to carrier frequency offset, resulting in InterCarrier Interference (ICI) [17].

\subsubsection{Inter-symbol Interference}

In telecommunication, Inter-Symbol Interference (ISI) is a 


\section{International Journal of Science and Research (IJSR) \\ ISSN (Online): 2319-7064}

Index Copernicus Value (2013): 6.14 | Impact Factor (2015): 6.391

form of distortion of a signal in which one symbol interferes with subsequent symbols. This is an unwanted phenomenon as the previous symbols have similar effect as noise, thus making the communication less reliable. ISI is usually caused by multipath propagation or the inherent non-linear frequency response of a channel causing successive symbols to "blur" together. In addition, if the modulation bandwidth exceeds the coherence bandwidth of the channel, ISI will be introduced and the consecutive transmitted symbols are distorted, since the past and current symbols of the signals overlap. Hence, at the receiver, channel equalizers have to be employed to remove the effects of ISI [10].

The presence of ISI in the system introduces errors in the decision device at the receiver output. Therefore, in the design of the transmitting and receiving filters, the objective is to minimize the effects of ISI, and thereby deliver the digital data to its destination with the smallest error rate possible [9].

\subsection{Channel estimation and equalization}

In digital communications, its purpose is to reduce InterSymbol Interference ISI to allow recovery of the transmit symbols. It may be a simple linear filter or a complex algorithm. The following equalizer types are commonly used in digital communications:

- Linear Equalizer: processes the incoming signal with a linear filter

- MMSE equalizer: designs the filter to minimize $E\left[|e|^{2}\right]$, where $e$ is the error signal, which is the filter output minus the transmitted signal. ${ }^{[1]}$

- Zero Forcing Equalizer: approximates the inverse of the channel with a linear filter.

- Soft Sphere Decoding: The output of an SSD is the loglikelihood ratios LLRs of the transmitted bits most likely to be involved in generation of the received signal.

The effect of an equalization systems to compensate for transmission channel impairments such as frequency dependent phase and amplitude distortion. Besides correcting for channel frequency-response anomalies, the equalizer can cancel the effects of multipath signal components, which can manifest themselves in the form of voice echoes, video ghosts or Rayleigh fading conditions in mobile communications channels. Equalizers specifically designed for multipath correction are often termed echo cancelers or deghosters. They may require significantly longer filter spans than simple spectral equalizers, but the principles of operation are essentially the same.

\subsection{Equalization techniques}

In this section we will express the mathematical description of the three equalization techniques that are investigated in this study.

\subsubsection{Minimum Mean Square Error (MMSE)}

A Minimum Mean Square Error (MMSE) estimator is a method in which it minimizes the mean square error (MSE), which is a common measure of estimator quality. Minimum mean-square error equalizer, which does not usually eliminate ISI completely but instead, minimizes the total power of the noise and ISI components in the output. The MMSE estimator is then defined as the estimator achieving minimal MSE. Generally, it very difficult to determine a closed form for the MMSE estimator; in these cases, one possibility is to seek the technique minimizing the MSE within a particular class, such as the class of linear estimators. The linear MMSE filter can be calculated as

$$
W=\left(H^{H} H+\sigma^{2} I_{M}\right)^{-1} H^{H}=G^{-1} H^{H} \text {, }
$$

Where $\mathrm{H}$ is the channel matrix, is the noise variance, (.) $\mathrm{H}$ is the complex conjugate transpose and IM is a $\mathrm{M} \times \mathrm{M}$ identity matrix. The received signal vector is then filtered to obtain the equalized signals as:

$$
\tilde{\chi}_{\text {MMSE }}=W_{y}
$$

Hence, the LMMSE detector minimizes the mean square error between the output $\tilde{X}_{\text {MMss }}$ and the transmitted signal (. The LMMSE filter can also be calculated using an extended channel matrix $\left(\mathrm{H} \in \mathbb{C}^{(\mathrm{M}+\mathrm{N}) \mathrm{x}}\right.$, as:

$$
\left.W=\underline{(H}^{H} \underline{H}\right)^{-1} \underline{H}^{H} \text {, }
$$

Where the extended channel matrix and received vector $\left(y \in \mathbb{C}^{\mathbb{C M I}+\mathrm{ND} \times \mathrm{l}}\right.$ can be expressed as:

$$
\underline{H}=\left[\begin{array}{c}
H \\
\sigma I_{N}
\end{array}\right] \text {, and } \underline{y}=\left[\begin{array}{c}
y \\
0 \\
N \times 1
\end{array}\right]
$$

Where $\quad 0_{\mathbb{N}}$ denotes a vector of zeros. The equalized signals can then be obtained from:

$$
\tilde{\chi}_{\text {MUSSE }}=\underline{H}^{+} \underline{y}
$$

Where (.)+ denotes the Moore-Penrose pseudo-inverse of the matrix [20]. This bias in the linear MMSE filter can be removed by $\mathrm{W}=\mathrm{BG}-1 \mathrm{HH}$ [19] where $\mathrm{B}$ is a diagonal matrix with (i,ith) element:

$$
B_{\mathrm{i}_{\mathrm{i}} \mathrm{i}}=\frac{p_{\mathrm{i}}+1}{p_{\mathrm{i}}}
$$

Then the SINR on stream (i) can be calculated as:

$$
p_{\mathrm{i}}=\frac{1}{\sigma^{2} G_{\mathrm{i} i \mathrm{i}}^{-1}}-1
$$

\subsubsection{Zero Forcing (ZF)}

The Least Square (LS) or Zero forcing (ZF) decoder is the simplest method used for decoding. The complexity is also the least. It is described in the following equation:

$$
G_{k}=\frac{1}{H_{k}}
$$

Where lis assumed to be the known channel. With the ZF equalizer, the interference is forced to zero, or assumed to be zero, which in turn amplifies the noise and can be seen from the following mathematical expression for OFDM if the first received symbol is expressed as and the second is expressed as :

$$
\hat{a}=x a+y b \cdot \text { and } \hat{b}=x b+y a
$$

The first equation shows that for the received symbol, the interference of received symbol $\mathbf{b}_{\text {is }}$ also available. So if one is to use the zero forcing of the equation above, then the

noise $\mathrm{n}$ is amplified. In case of MIMO detection, the $\mathrm{ZF}$ equalizer is given by:

$$
\mathrm{W}_{\mathrm{ZE}}=\left(\mathrm{H}^{\mathrm{H}} \mathrm{H}\right)^{-1} \mathrm{H}^{\mathrm{H}}=\mathrm{H}^{2}
$$

Where, ( is the channel matrix, and (is the pseudo inverse of the matrix [20]. The ZF receiver suppresses the 


\section{International Journal of Science and Research (IJSR) \\ ISSN (Online): 2319-7064}

Index Copernicus Value (2013): 6.14 | Impact Factor (2015): 6.391

interference between the MIMO streams, but it enhances the noise and the performance is far from optimal.

\subsubsection{Soft Sphere Decoding (SSD)}

In SSD, given the equation of MIMO and the MIMO channel modeling equation at a given time sample and finds the Maximum Likelihood for it.

$$
Y=H X+n
$$

Then the SSD finds the ML estimate for the transmitted modulated symbols $\widehat{\mathrm{X}}$ by :

$\widehat{\bar{X}}_{M L}=\arg \min \|Y-H X\|^{2}$

where $\mathrm{X} \in$ Eand $\Omega_{\text {is }}$ the complex-valued constellation from which the elements of $X$ are chosen. The SSD algorithm makes use of knowledge concerning the modulation scheme and the actual constellation and symbol mapping used in the modulator. It combines MIMO equalization and softdecision demodulation and maximizes the a posteriori probability measure to produce its output. The output of an SSD is the log-likelihood ratios LLRs of the transmitted bits most likely to be involved in generation of the received signal.

\section{Related Work}

The effect of fading and interference effects can be combated with equalizer for a MIMO system as mentioned in [21] and The BER characteristics for the various transmitting and receiving antennas simulated in MATLAB tool box and many advantages and disadvantages the system is described. An improved vertical Bell Labs Layered Space-Time (VBLAST) with spatial multiplexing technique used in MIMO systems is proposed in [22] with a Simulation results shows that the proposed V-BLAST, which employs SIC scheme, offers the performance very close to the optimal turbo MIMO approach, while providing stupendous improvements in computational complexity. In [23], a developed algorithms and architectures to meet the high data rate, low complexity requirements of the future mobile communication systems are proposed. With algorithms, architectures, and implementations for detection, channel estimation and interference mitigation in the multiple input multiple output (MIMO) orthogonal frequency division multiplexing (OFDM) receivers.

Beside the related issues mentioned in this section, we introduce the calculation of the Bit Error Rate (BER) to evaluate the performance of the Zero Forcing $\mathrm{ZF}$, the Minimum Mean Square Error MMSE and the Soft Sphere Decoding SSD. Also different types of modulations are considered such as QPSK, 16QAM, and 64QAM. The comparison is performed for different transmission modes such as SISO, SIMO, and MIMO.

\section{Methodology}

In order to study and evaluate a communication system performance with a specific assumptions and considerations using simulation environments we have to assume that system with the standard technical specification and regulations to ensure that our results are semi identical or close to real implementations in real world, so in this study we used the simulation environment of LTE as described in [30], which is implemented according to the standards of LTE that described in the technical specifications of the 3GPP.

The procedure that we followed here in order to gather the recorded results is described in the following flowchart.

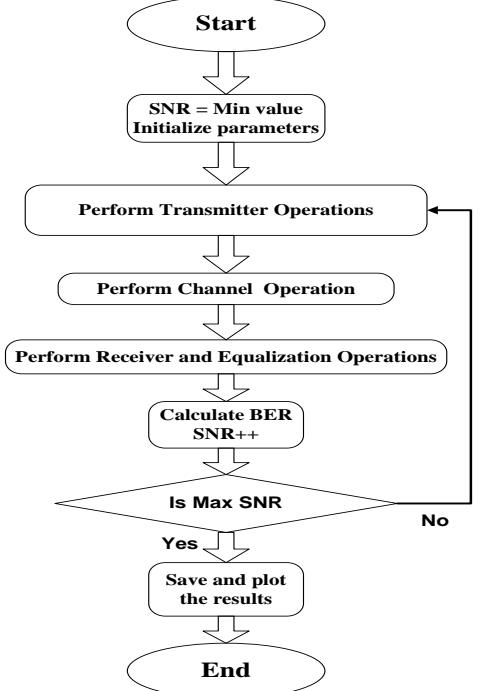

Figure 1: Simulation Flowchart

\section{System Model}

The system model here describes the physical layer procedure that implements the actual standard specification that is used in LTE for Downlink Shared channel DLSCH by deploying the Physical Downlink Shared channel PDSCH. The following figure shows the block diagram of the system model we assume in this study, and the different processing that are been used in LTE downlink model.

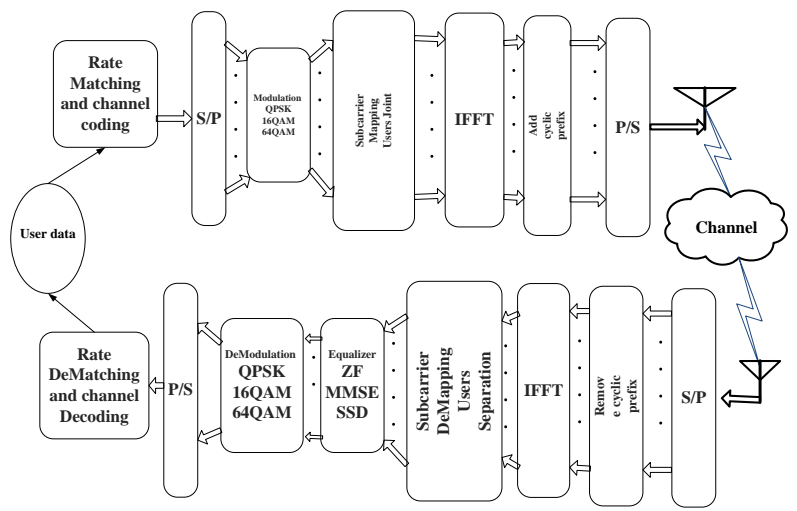

Figure 2: System block diagram

The system described above is then evaluated for different transmission modes such as SISO, SIMO, and MIMO. The MIMO scheme that used in LTE and considered in this study is the Open Loop Spatial Multiplexing OLSM, which refers to that the transmission, which is achieved with the minimum feedback.

\section{Volume 5 Issue 6, June 2016 www.ijsr.net}




\section{International Journal of Science and Research (IJSR) \\ ISSN (Online): 2319-7064}

Index Copernicus Value (2013): 6.14 | Impact Factor (2015): 6.391

Table 1: Simulation Parameters

\begin{tabular}{|c|c|}
\hline Parameter & Value \\
\hline Bandwidth & $10 \mathrm{MHz}$ \\
\hline Modulation & QPSK, 16QAM, 64QAM \\
\hline Coding & $1 / 3$ turbo coding \\
\hline Transmission modes & SISO, SIMO, MIMO-OLSM \\
\hline Channel & Frequency selective, high mobility \\
\hline Equalizers & ZF, MMSE, SSD \\
\hline Amount of simulated bits & $1 \mathrm{~Gb}$ \\
\hline SNR range & $-24-24$ \\
\hline
\end{tabular}

SIMO scheme used is $1 \times 4$, and $2 \times 2$ for MIMO. And also to be mentioned that for the SISO and SIMO transmission modes in LTE there are no actual implementation for the SSD equalizer, so the SSD is only used for MIMO spatial multiplexing.

\section{Results and Discussion}

Here the final results are figured, as mentioned before there are three main cases considered in this study, first in SISO transmission; figure (3) below shows the results of the ZF equalizer for different modulation schemes, and as expected higher modulation order such as 64QAM has the higher BER values, and lower modulation order has the lower BER.

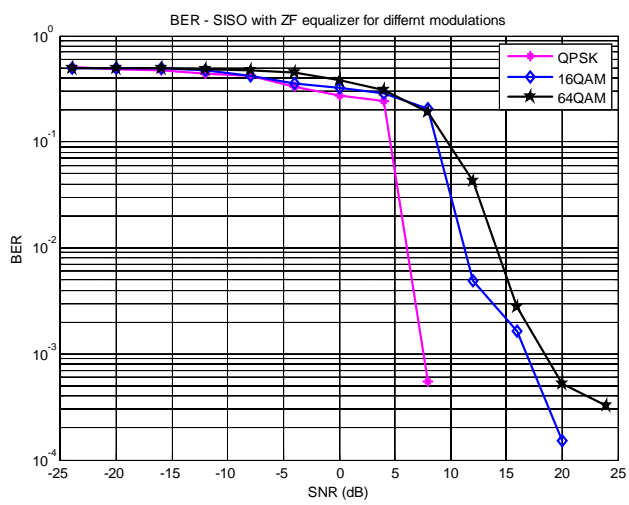

Figure 3: SISO BER for ZF equalizer

Figure (4) below also shows the BER of SISO but for the MMSE equalizer and for the three modulations, here also as the modulation order increases the BER increase, but we can figure that the MMSE BER values are eliminated with the lower values for SNR.

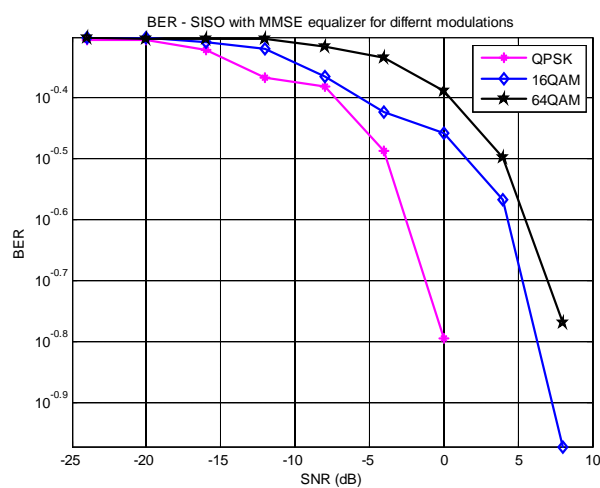

Figure 4: SISO BER for MMSE equalizer

Figure (5) below shows the BER of SISO for both equalizers, and for lower and higher modulations which are the QPSK and the 64QAM, this result proves that the MMSE equalizer is better than the $\mathrm{ZF}$ and this is obvious from the result below, as we can see that the BER of MMSE is eliminated before the $\mathrm{ZF}$, while the BER of ZF still occurs with higher SNR.

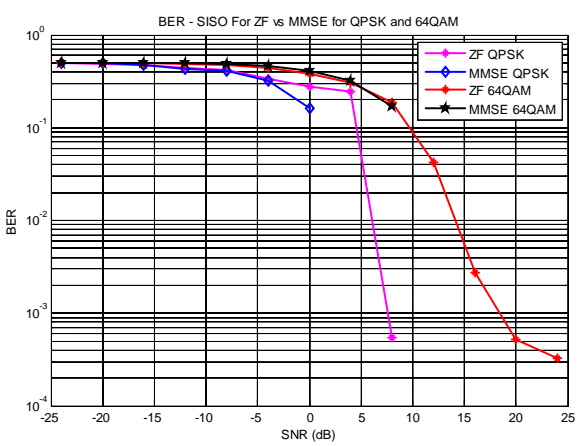

Figure 5: SISO BER for ZF and MMSE equalizer

The second case here is the SIMO transmission, the figure (6) here shows the BER of ZF equalizer of SIMO for the modulation schemes used, the results is the same and obvious that higher modulation orders achieves higher BER records.

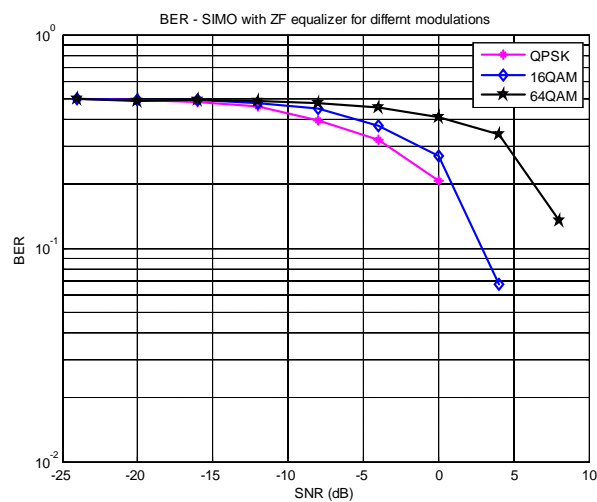

Figure 6: SIMO BER for ZF equalizer

Figure (6) here shows the BER of MMSE equalizer of SIMO with different modulations, here we can see that the performance of the both equalizers is approximately similar because BER is eliminated with earlier SNR values for both equalizers.

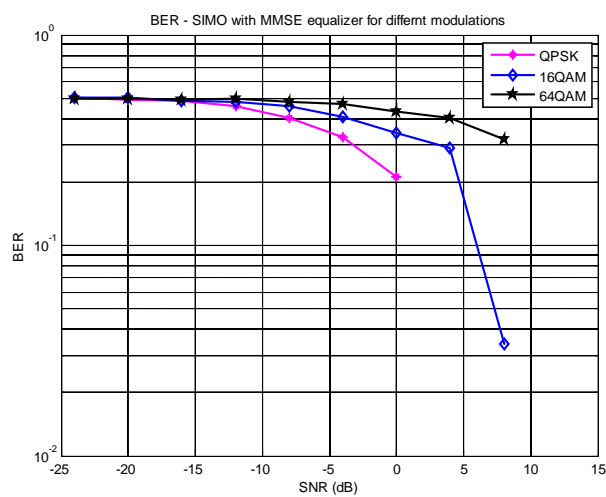

Figure 7: SIMO BER for MMSE equalizer

Figure (8) below shows the BER of SIMO for both equalizers, and for lower and higher modulations which are the QPSK and the 64QAM, here the ZF equalizer has the advantage over the MMSE in BER, and this proves that the complexity of the MMSE is affected by the number of receive antennas.

\section{Volume 5 Issue 6, June 2016 www.ijsr.net}




\section{International Journal of Science and Research (IJSR) \\ ISSN (Online): 2319-7064}

Index Copernicus Value (2013): 6.14 | Impact Factor (2015): 6.391

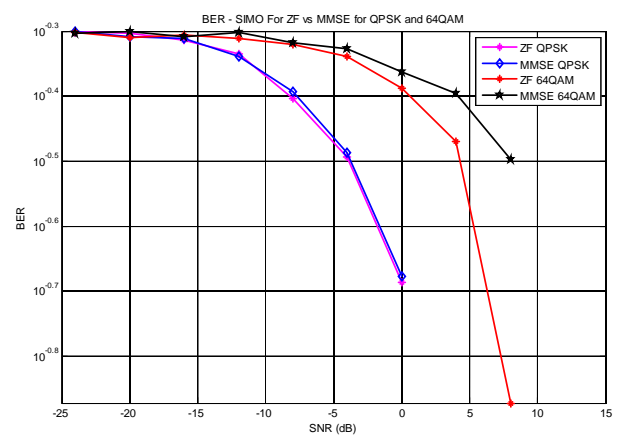

Figure 8: SIMO BER for MMSE and ZF equalizer

Finally, the third case is for MIMO transmission mode 3 of LTE which is the OLSM, here the use of the SSD is allowed and the results are reduced for only comparing the performance of the three equalizers for the highest modulation order. Figure (9) below shows that the ZF equalizer has the Higher BER values, the MMSE has average BER records, while the SSD performs better than both equalizers, so the SSD is the best equalization technique in our case.

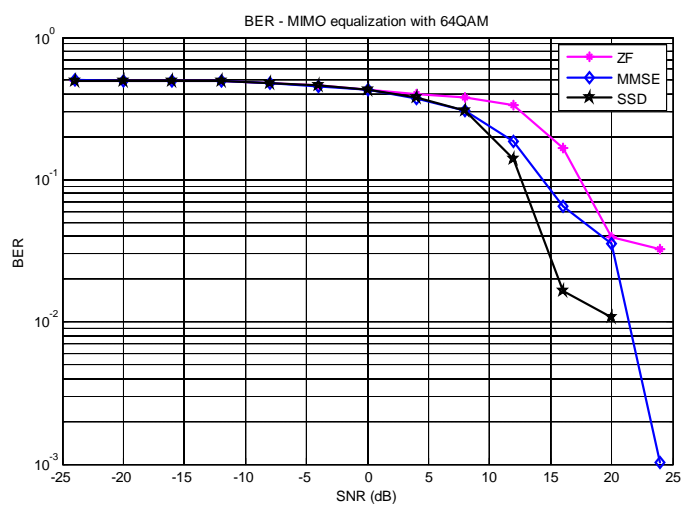

Figure 9: SIMO BER for MMSE and ZF equalizer

\section{Conclusion}

There are two familiar equalizers are verified by this study, the Zero forcing (ZF) and the Minimum Mean Square Error (MMSE), the ZF is meant by bushing the error value to zero, the MMSE minimize the mean square error value. In addition to the Soft Sphere Decoding (SSD) equalizer which is originally added for the LTE in MIMO transmission mode 3.

From the simulation we realize that the MMSE has better results than the $\mathrm{ZF}$ equalizer in case of single antennas configuration SISO and in MIMO, but the ZF equalization has the advantage in case of SIMO, in MIMO case the three equalizers are compared and the results shows that the SSD equalizer has the advantage over the ZF and the MMSE in OLSM or LTE transmission mode 3.

\section{References}

[1] 3GPP LTE Encyclopedia. "An Introduction to LTE"., (accessed April 3, 2016).

[2] Penttinen, J. (2011) The LTE/SAE Deployment Handbook, John Wiley \& Sons, Ltd, Chichester.
[3] Holma, H. and Toskala, A. (2011) LTE for UMTS: Evolution to LTE-Advanced, Ch. 10, John Wiley \& Sons, Ltd, Chichester.

[4] 3GPP TR 36.814 (March 2010) Further Advancements for E-UTRA Physical Layer Aspects, Release 9, annex

[5] European Cooperation in Science and Technology (1999) Digital Mobile Radio Towards Future Generation System, COST 231 final report. Available at: http://www.lx.it.pt/cost231/final_report.htm (28 Apr. 2015).

[6] 3GPP TS 25.306 UE Radio Access Capabilities, Release 10 , section 5

[7] 3GPP TS 36.306 User Equipment (UE) Radio Access Capabilities, Release 10, section 4.1

[8] 3GPP TS 36.101 User Equipment (UE) Radio Transmission and Reception, Release 10, section 5.6A

[9] Wikipedia contributors. "Intersymbol interference." Wikipedia, The Free Encyclopaedia. Wikipedia, The Free Encyclopaedia, 25 Jun. 2014. Web. 28 Apr. 2015

[10] L. Hanzo, T. H. Liew, and B. L. Yeap, Turbo Coding, Turbo Equalisation and Space-Time Coding. Chichester: IEEE Press and John Wiley \& Sons, Ltd, 2002. http://www-mobile.ecs.soton.ac.uk.

[11] Holma, H. and Toskala, A. (2011) LTE for UMTS: Evolution to LTE-Advanced, Ch. 10, John Wiley \& Sons, Ltd, Chichester.

[12]L. Hanzo, W. Webb, and T. Keller, "Single- and Multicarrier Quadrature Amplitude Modulation". Chichester: IEEE Press and John Wiley \& Sons, Ltd, 2nd edn, 2000.

[13] L. Hanzo, M. M“unster, B. J. Choi, and T. Keller, "OFDM and MC-CDMA for Broadband Multi-user Communications, WLANs and Broadcasting". Chichester: IEEE Press and John Wiley \& Sons, Ltd, 2003.

[14] L. Hanzo, J. Akhtman, L. Wang, M. Jiang. "MIMOOFDM for LTE, WiFi, and WiMAX : coherent versus non-coherent and cooperative turbo-transceivers". John Wiley \& Sons Ltd, 2011.

[15] Institute of Electrical and Electronics Engineers, IEEE Standard 802.16: Air Interface for Fixed Broadband Wireless Access Systems, 2004.

[16]R. Bercovich, 'OFDM enhances the $3 \mathrm{G}$ high-speed data access', in GSPx 2004 Conference,(Santa Clara, USA), 27-30 September 2004 http://www.techonline.com/pdf/pavillions/gspx/2004/10 84.pdf.

[17] L. Hanzo and B. Choi, 'Near-instantaneously adaptive HSDPA-style OFDM and MC-CDMA transceivers for WiFi, WiMAX and next-generation systems', Proceedings of the IEEE, vol. 95, pp. 2368-2392, December 2007.

[18] A. J. Paulraj, D. A. Gore, R. U. Nabar, and H. B“olcskei, 'An overview of MIMO communications - a key to gigabit wireless', Proceedings of the IEEE, vol. 92, pp. 198-218, February 2004.

[19]Collings I, Butler M \& McKay M (2004), "Low complexity receiver design for MIMO bit-interleaved coded modulation. Proc. IEEE Int. Symp. Spread Spectrum Techniques and Applications". Sydney, Australia.

[20] Hassibi B (2000) A fast square-root implementation for BLAST. Proc. Annual Asilomar Conf. Signals, Syst., Comp. Asilomar, USA, vol. 2, 1255-1259. 


\section{International Journal of Science and Research (IJSR) \\ ISSN (Online): 2319-7064}

Index Copernicus Value (2013): 6.14 | Impact Factor (2015): 6.391

[21] VinayDawar, Ritu Sharma, "Reduction in Bit Error Rate from Various Equalization Techniques for MIMO Technology", International Journal of Soft Computing and Engineering, Volume-2, Issue-4, September 2012.

[22] SwetamadhabMahanta, AnkitRajauria, "Analysis of MIMO System Through ZF \& MMSE Detection Scheme", IJECT Vol.4, Issue spl-4, April-June 2013.

[23] Ketonen, Johanna, "Equalization and channel estimation algorithms and implementations for cellular MIMOOFDM downlink", Acta Univ. Oul. C 423, 2012.

[24] VineethaMathai, K. Martin Sagayam, "Comparison And Analysis Of Channel Estimation Algorithms In OFDM Systems" IJSTR vol 2, Issue 3, March 2013.

[25]3GPP TS 36.141 V12.7.0, "Evolved Universal Terrestrial Radio Access (E-UTRA) Base Station (BS) conformance testing" (Release 12).

[26]3GPP TS 36.101 V12.7.0 "Evolved Universal Terrestrial Radio Access (E-UTRA) User Equipment (UE) radio transmission and reception", (2015-03).

[27] Pätzold, Matthias, Cheng-Xiang Wang, and Bjørn Olav Hogstad. "Two New Sum-of-Sinusoids-Based Methods for the Efficient Generation of Multiple Uncorrelated Rayleigh Fading Waveforms." IEEE Transactions on Wireless Communications. Vol. 8, 2009, Number 6, pp. 3122-3131.

[28] Dent, P., G. E. Bottomley, and T. Croft. "Jakes Fading Model Revisited." Electronics Letters. Vol. 29, Number 13, pp. 1162-1163.

[29]3GPP TS 36.104. "Base Station (BS) radio transmission and reception." 3rd Generation Partnership Project; Technical Specification Group Radio Access Network; Evolved Universal Terrestrial Radio Access (E-UTRA).

[30] Zarrinkoub, Houman, (2014)," Understanding LTE with MATLAB : from mathematical foundation to simulation, performance evaluation and implementation”, John Wiley \& Sons, Ltd. 